\title{
The Numerical Simulation of Unsteady Flow in a Mixed flow Pump Guide Vane
}

\author{
Li Yi-bin, Li Ren-nian and Wang Xiu-yong \\ School of Energy and Power Engineering, Lanzhou University of Technology \\ No.287 Langongping Road, Lanzhou, 730050, China, liyibin58@163.com
}

\begin{abstract}
In order to investigate the characteristics of unsteady flow in a mixed flow pump guide vane under the small flow conditions, several indicator points in a mixed flow pump guide vane was set, the three-dimensional unsteady turbulence numerical value of the mixed flow pump which is in the whole flow field will be calculated by means of the large eddy simulation (LES), sub-grid scale model and sliding mesh technology. The experimental results suggest that the large eddy simulation can estimate the positive slope characteristic of head \& capacity curve. And the calculation results show that the pressure fluctuation coefficients of the middle section in guide vane inlet will decrease firstly and then increase. In guide vane outlet, the pressure fluctuation coefficients of section will be approximately axially symmetrical distribution. The pressure fluctuation minimum of section in guide vane inlet is above the middle location of the guide vane suction surface, and the pressure fluctuation minimum of section in which located the middle and outlet of guide vane. When it is under the small flow operating condition, the eddy scale of guide vane is larger, and the pressure fluctuation of the channel in guide vane being cyclical fluctuations obviously which leads to the area of eddy expanding to the whole channel from the suction side. The middle of the guide vane suction surface of the minimum amplitude pressure fluctuation to which the vortex core of eddy scale whose direction of fluid's rotation is the same to impeller in the guide vane adhere.
\end{abstract}

Keywords: Mixed-flow Pump, Guide Vane, Pressure fluctuation, Vortex, LES, CFD

\section{Introduction}

The specific speed $n_{\mathrm{s}}$ of mixed flow pump is between 300 and 600 whose performance is between centrifugal pump and axial-flow pump. Its applications are extended to the field of centrifugal pump and axial-flow pump. Because of the outstanding merits of compact structure, easy to start and high efficiency, mixed-flow pump has been applied to desalination unit, power plant and nuclear power water cycle system.

At present, the pressure fluctuation of the unsteady flow in mixed flow pump is one of the main reason that cause pump unit vibration and noise. With the wider application of mixed flow pump unit, the problems of unstable operation which are caused by the hydraulic excitation have become increasingly prominent. Due to the high price, time costing and lag of the experimental method, and with the development of fluid dynamics, CFD (Computational Fluid Dynamics) numerical calculation technology has been widely used in the field of three dimensional turbulence numerical simulation with advantage of short period and leading character. At present, the turbulence numerical simulation of a mixed-flow pump widely adopts the Reynolds time average time method, because of the problem of unsteady flow, there being drawbacks in the Reynolds equation and turbulence model. While LES (large eddy simulation) method has specific superiority in simulating the unsteady pressure fluctuation of hydraulic machinery flow field. In China, Wang Fu-jun et.al. ${ }^{[1]}$ study the flow characteristics of the unsteady and unstable flow using LES subgrid turbulence model. Abroad Kato ${ }^{[2]}$ study the unsteady flow characteristics of mixed flow pump under off-designed condition based on LES sub-grid turbulence model. Miorini, et.al. ${ }^{[3]}$ discover the blade tip leakage vortex structure and unsteady flow mechanism of mixed-flow pump by PIV (Particle Image Velocimetry) and CFD (Computational Fluid Dynamics). Miyabe ${ }^{[4]}$ reveals the unstable operation characteristic caused the large-scale backflow between guide vane inlet and impeller outlet under small flow conditions by extracting the test signal of pressure fluctuation. Byskov, et.al. ${ }^{[5]}$ simulate the internal flow of centrifugal pump impeller using LES, and the calculational results being compared with the Reynolds time average time method and experimental data. It is believed that the LES can show the complicated flow phenomena inside centrifugal impeller. Nagahara, et.al. ${ }^{[6]}$ do numerical research on inner flow field and pressure fluctuation of flow passage components in a multi-stage centrifugal

Received September 7 2012; revised January 21 2013; accepted for publication February 28 2013: Review conducted by Prof. Hyung-Hee Cho. (Paper number O13011S) 
pump by using LES, who also point out that the spectrum trend of pressure fluctuation is in accordance with experiment basically. Doing unsteady flow numerical simulation of inner flow field in the mixed flow pump can forecast the characteristics of pressure fluctuation through analyzing the complexity of mixed flow pump inner flow field by using numerical simulation, which has great significance for the project.

The LES was used in the calculation of full-flow road, three dimensional unsteady turbulence of mixed flow pump, while external characteristic of the predicted performance were compared with the experimental results to verify the reliability. Then the flow law in eddy scale of guide vane section and the pressure fluctuation characteristics of indicator points in the impeller are analyzed.

\section{Calculation model and numerical method}

This paper selects a mixed flow pump model with flow rate $Q=490 \mathrm{~m}^{3} / \mathrm{s}$, head $H=9.5 \mathrm{~m}$ and the rotating speed $n$ $=1450 \mathrm{r} / \mathrm{min}$. The geometry parameters are that the impeller outlet diameter $D_{2}=290 \mathrm{~mm}$, the suction chamber diameter $d=220 \mathrm{~mm}$, the impeller blade number is 4 and the blade number of guide vane is 7 .

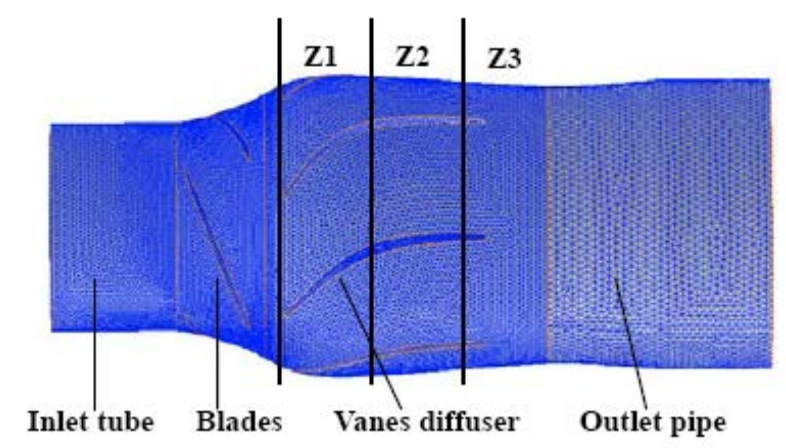

Fig. 1 Mixed flow pump calculating domain and grid

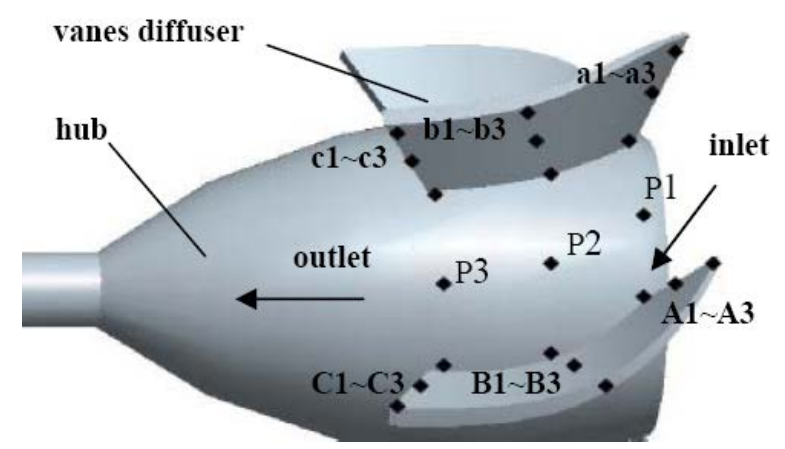

Fig. 2 Indicator points in the mixed-flow pump

The calculational domain includes inlet tube, blades, guide vane and outlet pipe. The calculating mesh is consisting of the unstructured grid and structured grid. The regional grid of impeller and guide vane is refined and the total unit is 1860,250 so as to improve the calculating accuracy. In order to obtain the most economical number of grid and calculating step, the gridindependence and calculating step-independence are checked.

Turbulence model adopts LES Smagorinsky-Lilly subgrid scale model ${ }^{[7]}$, Smagorinsky constant $C_{s}=0.1$, which can be used in the majority of fluid conditions. The transient control equations are dispersed by finite volume method, solved by using the coupling implicit scheme, the relative fluid project being dispersed by second order upwind scheme, and adopting the more effective PRESTO difference scheme ${ }^{[8]}$ of the high reynolds number of high strong vortex to disperse the pressure project. The unsteady calculational PISO algorithm ${ }^{[9]}$ (is been used to solve pressure and speed coupling. The location of inlet boundary is selected in flange of pump inlet where the speed is named as the condition of entrance, whose value is determined by the flow and area. Outlet boundary lies in the flange of pump outlet, the outflow condition being pointed, assuming that the flow in the pump has reached full development state. Sliding mesh technology is introduced into dealing with the interface between rotor and stator of the pump. And the result of steady state RANS calculation is regarded as the initial flow field, while the wall in a condition of no-slip, aiming at distinguishing the information of inner flow field by the means of fixing the time step is $1 / 120$ of runner's rotation, and actual time step $\Delta t$ is $3.4483 \times 10^{-4} \mathrm{~s}$.

Three indicator interfaces are set in guide vane axial direction, which are guide vane inlet indicator interface $Z_{1}$, middle indicator interface $Z_{2}$ and outlet indicator interface $Z_{3}$. For the purpose of surveying the characteristics of pressure fluctuations in a mixed-flow pump guide vane, several indicator points are set in which are pressure interface with $A_{1}$ to $A_{3}, B_{1}$ to $B_{3}$, and $C_{1}$ to $C_{3}$, suction interface with $a_{1}$ to $a_{3}, b_{1}$ to $b_{2}$,and $c_{1}$ to $c_{3}$,and hub interface with $P_{1}, P_{2}, P_{3}$. The indicator points are showed in Fig.2. 


\section{Result and discussion}

\subsection{Performance prediction}

According to the dynamic performance prediction method of mixed flow pump, the unsteady average time characteristics of the pump can be acquired. While the performance prediction of mixed flow pump head under 6 kinds of operating condition is being compared with the test result, the abscissa is relative capacity $Q / Q_{d}$ and the ordinate is relative head $H / H_{d}$ which are showed in Fig .3.

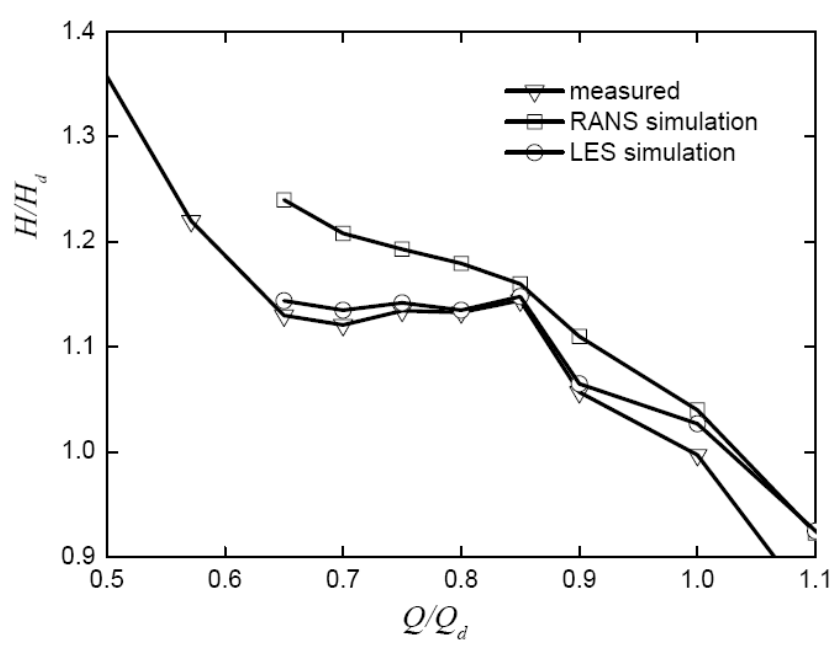

Fig. 3 Performance prediction and experimental verification.

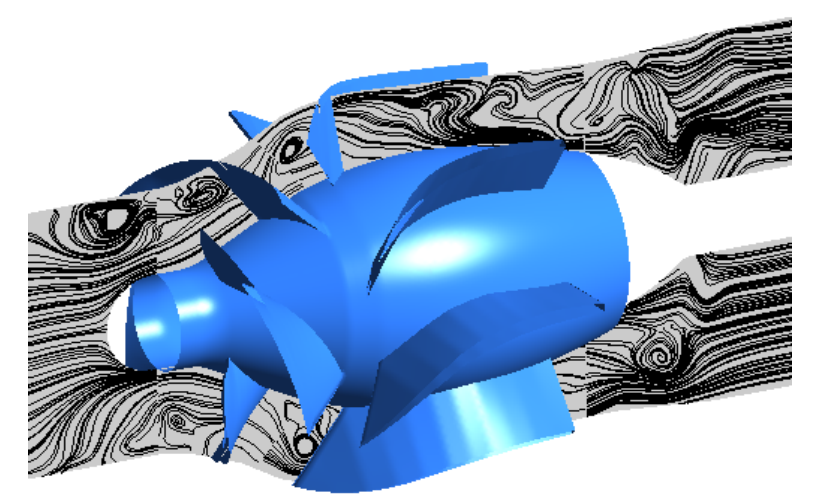

Fig. 4 Streamline of meridian plane under $0.6 Q_{\text {opt }}$ operating condition

Experimental result is in keeping with prediction result, and the heads of all the points comparing to the predictive efficiency with a relative error of lower than $4 \%$, during experimental analysis, the existence of $H$-Q positive slop between $0.6 Q_{o p t}$ and $0.85 Q_{o p t}$. There have been large-scale backflow and secondary flow appearing in mixed flow pump impeller and guide vane channel under the $0.6 Q_{\text {opt }}$ operating condition, which mean that there exists a large-scale stalling region. Comparing to the prediction of Reynolds-averaged Navier-Stokes (RANS)equations, the CFD unsteady performance prediction cannot forecast the $H$-Q positive slope precisely basing on the equations. The research conclusion of $H-Q$ satisfies the result of large eddy simulation and sub-grid turbulence model prediction in the range of $0.68 Q_{o p t}$ to $0.87 Q_{o p t}$. So the calculation of large eddy simulation has higher reliability.

\subsection{Analysis of pressure fluctuation characteristics}

Pressure coefficients is defined as

$$
C_{p}=\frac{p-\bar{p}}{\frac{1}{2} \rho u_{2}^{2}}
$$

Where $p$ is static pressure of indicator points; $\bar{p}$ is average static pressure in a rotating cycle; $u_{2}$ is circumference velocity of impeller outlet.

Comparing pressure fluctuation coefficients of indicator points in $Z_{1}, Z_{2}$ and $Z_{3}$ section basing on the Fig.5, Fig.6 and Fig.7, there is no apparent fluctuations in $Z_{1}$ and the coefficients decreasing firstly then increasing along the direction of pressure surface, hub and suction interface. In $Z_{3}$, the coefficients symmetrically distribute centering the middle part of two adjacent guide vanes. Under $Q_{\text {opt }}$ operating condition in $Z_{3}$ section, the pressure fluctuation coefficients are evenly distributed along circumference. All in all, the coefficients distribute evenly along circumference under the three kinds of conditions. When it is under $0.6 Q_{\text {opt }}$ working mode, the fluctuation amplitude is largest, in contrast to $Q_{o p t}$ whose amplitude is smallest. The minimum value of pressure fluctuation is locating in $Z_{1}$, while they are $P_{2}$ and $P_{3}$ in $Z_{2}$ and $Z_{3}$. 


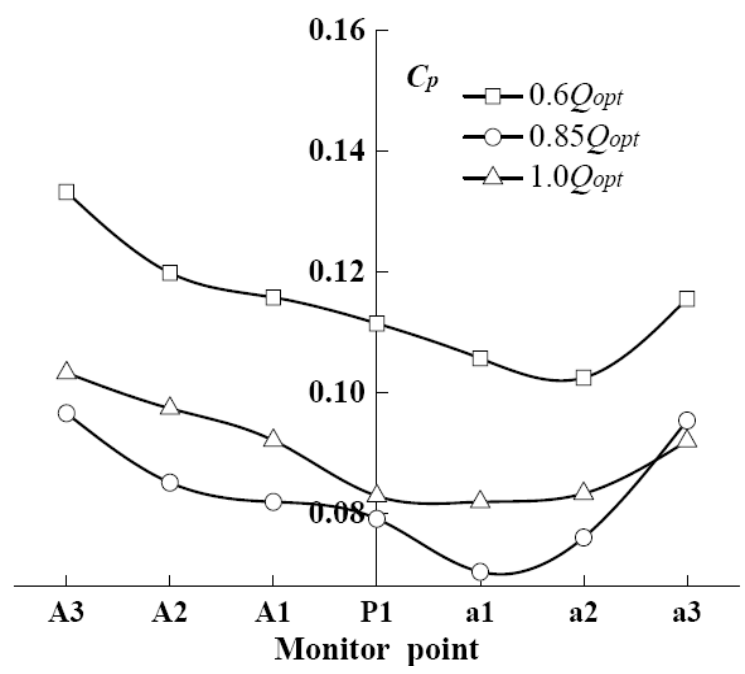

Fig. 5 Pressure coefficients of indicator points in Z1 section

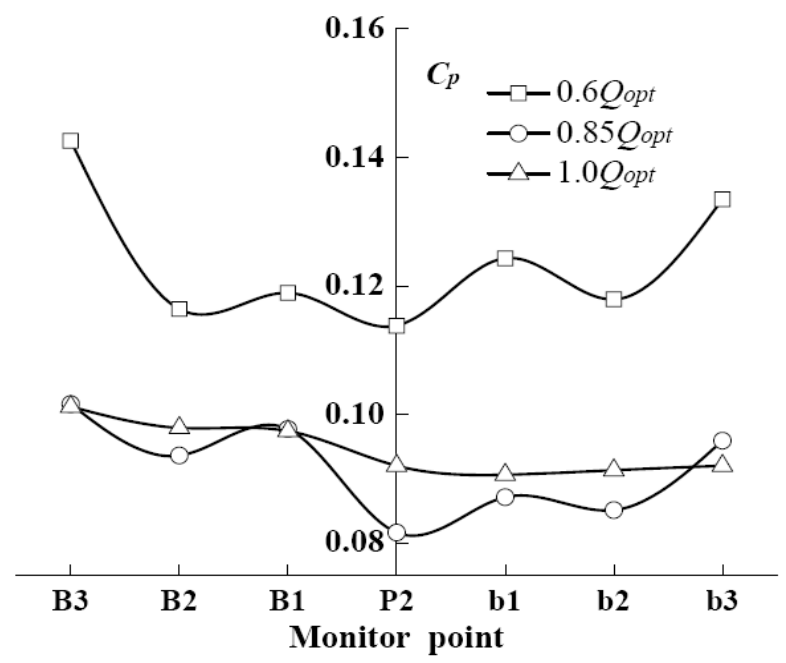

Fig. 6 Pressure coefficients of indicator points in Z2 section

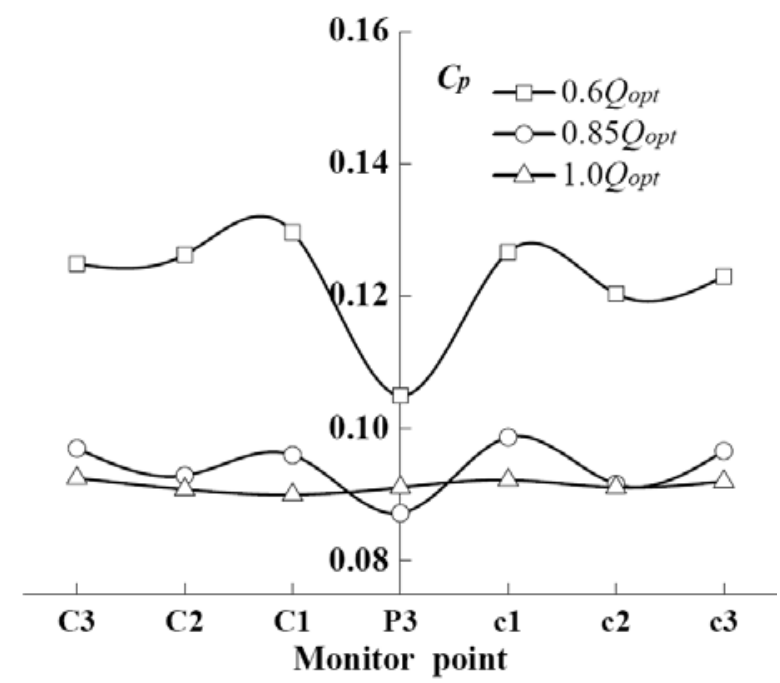

Fig. 7 Pressure coefficients of indicator points in Z3 section.

\subsection{Analysis on streamlines of guide vane characteristic sections}

Under $0.6 Q_{\text {opt }}$ operating condition, there being a large scale of vortex area in the meridian channel, whose liquid flow energy dissipation is decided by the scale and strength. Due to the disturbance and structure of impeller and guide vane reducing the scale of vortex, the appearance of vortex area covering the impeller and the whole channel of guide vane, the scale becoming larger and 
larger in the part of inlet and outlet and the vortex area being reduced in the channel which can be learned from Fig.4. When the vortex area block the channel, the sudden acceleration and deceleration of liquid will change the speed and direction with producing new vortex sometime. The transformation of liquid velocity vector is named as rotating stall which happens in the channel of impeller and guide vane with increasing hydraulic loss.

From the $0.6 Q_{o p t}, 0.85 Q_{o p t}$, and $Q_{o p t}$ operating condition, the vortex scale of section $Z_{1}$ is decreasing until disappeared gradually what is also appearing in the section $Z_{2}$. After comparing the vortex of section $Z_{1}, Z_{2}$ and $Z_{3}$, what is confirmed is that the scale of guide vane is larger under small flow conditions. The vortex area which increases firstly then decreases from inlet to outlet channel is small under optimum condition.

As a result of the side static pressure value of guide vane suction interface in $\mathrm{Z} 1$ section, the vortex area adheres to side channel. While the amplitudes of pressure fluctuation indicator points symmetrically distribute in Z2 and Z3 section channels, a cyclical swing is appearing which leads to the vortex area covering the whole channel from the side suction, and the vortex core sticking to the indicator area of a2, b2 and c2, besides the rotating directions of vortex area being consistent with impeller, which are showed in Fig. 8.and Fig 9.

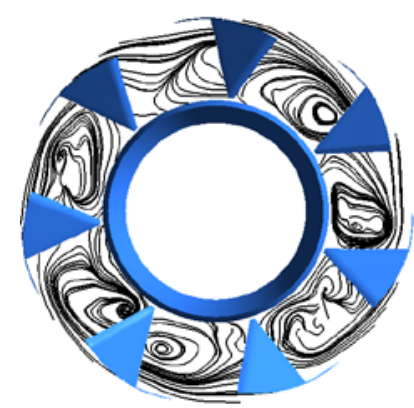

(a) $0.6 Q_{\text {opt }}$

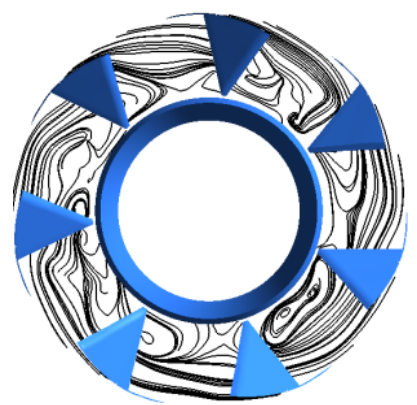

(b) $0.85 Q_{\text {opt }}$

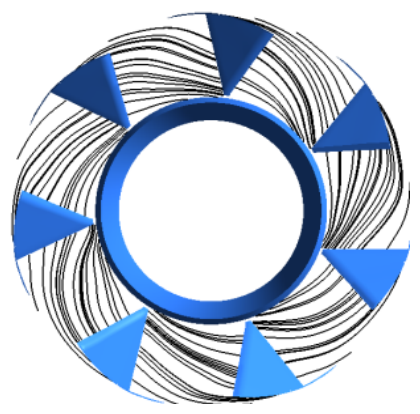

(c) $Q_{\text {opt }}$

Fig. 8 Streamline of indicator points in Z1 section

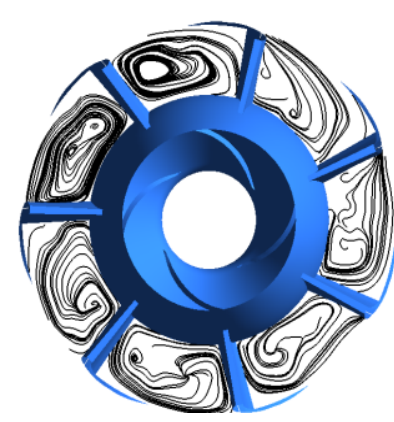

(a) $0.6 Q_{\text {opt }}$

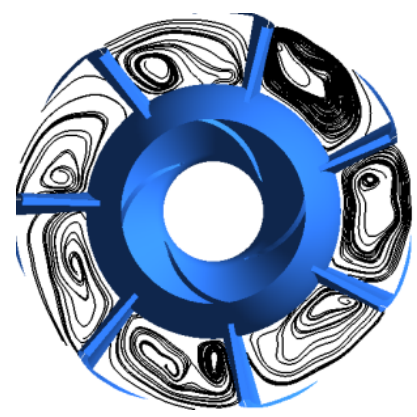

(b) $0.85 Q_{o p t}$

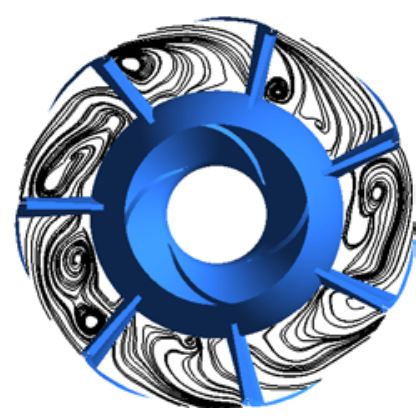

(c) $Q_{\text {opt }}$

Fig. 9 Streamline of indicator points in $\mathrm{Z} 2$ section

\section{Conclusion}

On account of the unsteady calculating simulation of mixed flow pump with using LES and sub-grid scale model, and comparing the prediction with experimental results, LES can predict the positive slope of head-capacity curve precisely. And the unsteady pressure fluctuation characteristic of mixed flow pump is analyzed basing on the previous research.

The pressure fluctuation coefficients of $Z_{1}$ and $Z_{2}$ decrease firstly and then increase accordingly. In $Z_{3}$ section, the coefficients symmetrically distribute centering the hub. Under $Q_{o p t}$ operating condition in $Z_{3}$ section, the pressure fluctuation coefficients are evenly distributed along circumference. Under the three kinds of conditions the coefficients distribute evenly along circumference. The minimum value of pressure fluctuation is locating in guide vane which is above the middle of suction interface in $Z 1$. While in $Z_{2}$ and $Z_{3}$, the minimum is the middle of the hub in $Z_{2}$ and $Z_{3}$.

Under small flow conditions the scale of guide vane is larger. The vortex area which increases firstly then decreases from inlet to outlet channel is small under optimum conditions. A cyclical swing is appearing which leads to the vortex area covering the whole channel from the side suction, and the vortex core sticking to the guide vane which is above the middle of suction interface, besides the rotating directions of vortex area being consistent with impeller.

\section{Acknowledgments}

This work is financially supported by the National Natural Science Fund Project (Project Nos.51369015) and supported by the Gansu Province Natural Science Fund Project (Project Nos.2011GS04264) 


\section{Nomenclature}

$Q \quad$ Flow rate $\left[\mathrm{m}^{3} / \mathrm{s}\right]$

$Q_{d} \quad$ Flow rate at design condition $\left[\mathrm{m}^{3} / \mathrm{s}\right]$

$Q_{\text {opt }} \quad$ Flow rate at optimal condition $\left[\mathrm{m}^{3} / \mathrm{s}\right]$

$H \quad$ Head[m]

$H_{d} \quad$ Head at design condition [m]

$n \quad$ Rotating speed [ $\mathrm{r} / \mathrm{min}]$

$D_{2} \quad$ Impeller outlet diameter [mm] $d \quad$ Suction chamber diameter [mm]

$\Delta t \quad$ Time step $[\mathrm{s}]$

$u_{2} \quad$ Circumference velocity of impeller outlet $[\mathrm{m} / \mathrm{s}$ ]

$p \quad$ Static pressure $[[\mathrm{kPa}]$

$\bar{p} \quad$ Average static pressure $[\mathrm{kPa}]$

$\rho \quad$ Fluid density $\left[\mathrm{kg} / \mathrm{m}^{3}\right]$

$C_{p} \quad$ Pressure coefficient

\section{References}

[1] Wang F.-J., Zhang L., Zhang Z.-M, 2007, “Analysis on pressure fluctuation of unsteady flow in axial flow pump,” Shui LI Xue Bao, 38(08):1003 1009.

[2] Kato C., et al. 2003, “Large-Eddy Simulation of Unsteady Flow in a Mixed How Pump,” International Journal of Rotating Machinery, Vol. 9, pp. 345 351.

[3] Rinaldo L. M., Wu H.-X.,and Joseph K., 2010, “The internal structure of the tip leakage vortex within the rotor of an axial water-jet pump,” Proceedings of ASME Turbo Expo: Power for Land, Sea and Air GT2010. June 14 18, Glasgow, UK, pp. 1 16.

[4] Miyabe M., Maeda H., Umeki I., et al. 2006, “Unstable head-flow characteristic generation mechanism of a low specific speed mixed flow pump,” Journal of Thermal Science, 1(2): 115 120.

[5] Byskov R. K., Jacobsen C. B., Pedersen N., 2003, Flow in a centrifugal pump impeller at design and off-design conditions, part II: large eddy simulations,” J. of Fluids Eng., Trans. of the ASME, 125(1): 73 83,

[6] Takahide N., Yasuhiro I., and Toshiyuki S., et al, 2005, "Investigation of the flow field in a multistage pump by using LES," Proceedings of 2005 ASME Fluids Eng., Division Meeting, FEDSM2005, Houston, TX, United States, pp. 1476 1487.

[7] Smagorinsky J., 1963, “General circulation experiments with the primitive equations-I: the basic experiment," Monthly Weather Review, 91(3):99 164.

[8] Slack M. D., Prasad R. O., and Bakker A., et al.2000, “Advances in cyclone modeling using unstructured grids,” Chemical Eng., Research and Design, 78(A8): 1098 1104.

[9] Issa R. I., 1986, "Solution of implicitly discretized fluid flow equations by operator splitting," Journal of Computational Physics, 62:40 65. 